\section{Origin of Cross-Cousin Marriage in Assam}

Cross-cousin marriage is widely prevalent among the primitive peoples of Assam. Of the two types, the marriage with the daughter of the mother's brother is more frequent. Different theories have been put forward by ethnologists as to the origin of this custom, but none of them seems to be tenable in Assam area.

Though most of the tribes of Assam are now patrilineal, yet an earlier stratum of matriliny should not be overlooked. Even among some of the tribes, matrilineal traits are still dominant. These matrilineal people probably came in contact with later patrilineal immigrants of superior culture, and marriages between them were frequent. During this period, the immigrant people, in order to give their sister's family better economic status, introduced the marriage of their sister's son to their own daughters. The contact in some cases led to the formation of dual groups, and there both types of cross-cousin marriage were permitted. But after a time, when the domination of the patrilineal people was complete, the avoidance of the father's sister's daughter came from the idea that father and father's sister inherited the same blood, so her daughter, who also inherited a part of the blood through her mother, was not marriageable. This idea is still common among the Chothe Kukis, Mantak Koms and other tribes of Assam. In some cases, the patrilineal dominance is so strong that, in the absence of the mother's brother, the bride-price accrues to his son, that is, the mother's brother's son will get the payment for his father's sister's daughter, as is found among the Chothes.

This rule, on the face of it, seems to be absurd, but if we go into details we can easily understand the underlying principle. In the early matrilineal stage referred to, the marriage between the children of brothers and sisters was not looked upon with favour by the earliest group as it would break the 'household exogamy'. But the later group would not observe this rule, as it would be against their interest, and they might have no reason for observing 'household exogamy'. The husband in such a group, finding property to descend matrilineally, would try to provide for his portionless sister's family by marrying his sister's son to his daughter. The wife who owns the property and observes the rules of the earlier people would not object as 'household exogamy' on her side of the family would not be violated. In this way marriage with the daughter of the mother's brother would come into vogue. But she would be opposed to her children marrying her own brother's children. Hence the other cross-cousin marriage (father's sister's daughter) would not be permissible. In some cases also, marriage with the daughter of the mother's brother would not be allowable. But if the three groups (two older and the one later) were linked up in a tri-clan system, this problem would disappear.

If later on, through the dominance of the patrilineal people of this group or other groups, the whole social and economic structure passes on to fatherright, only the tradition of such preferential mating, with occasional actual marriages, would persist. This seems to be the present position in Assam.

\section{J. K. Bose.}

Department of Anthropology, University, Calcutta.

Dec. 31 .

\section{Annual Variation of the Critical Frequencies of the $E$ - and $F_{2}$-Layers}

The successive accumulation of data on the critical frequencies, that is, the limiting penetrating frequencies, of the $E$ - and $F$-layers determined from noon observations show the following main features ${ }^{1}$ : the critical frequencies of the $E$-layer show a regular annual variation depending on the height of the sun, whereas the critical frequencies of the $F_{2}$-layer show an inverse annual variation, with high values during winter and lower values in summer. Appleton ${ }^{2}$ has explained this as a temperature effect, the density of the air at $200-400 \mathrm{~km}$. in winter being higher on account of the lower temperature. According to the simple theory of photo-ionization, the maximum electron density, that is, the critical frequency, will depend on the pressure.

From a geophysical point of view, it is difficult to understand why an annual temperature variation should be restricted to the $F_{2}$-layer alone. Studies of the variation of the auroral heights in the border region between the dark and sunlit atmosphere have shown ${ }^{3}$ that there is a marked diurnal variation of temperature at a height of $100 \mathrm{~km}$., that is, in the region of the $E$-layer. It is therefore probable that the annual variation of temperature in the $F_{2}$-layer also must include the $E$-layer.

A hypothesis explaining the dependence of the critical frequencies of the $F_{2}$-layer on, and the independence of the critical frequencies of the $E$-layer of, the annual temperature (and therefore, pressure) variations will be put forward in the following, based on noon observations of the critical frequencies recorded at Tromso $\left(\varphi=69 \cdot 7^{\circ} \mathrm{N} ., \lambda=18 \cdot 9^{\circ} \mathrm{E}\right.$. $)$ during the last two years.

The observations from Tromso show the same inverse annual variation of the critical frequencies of the $F_{2}$-layer with high values in winter and low values in summer previously observed. During the dark period in winter, when the sun's height at noon is negative, the sun's rays when producing the dense $F_{2}$-ionization in the zenith have to enter the layer from below after first having passed once through the layer. During the same period one does not obtain $E$-echoes on undisturbed days. This means that the part of the solar spectrum producing the $F_{2}$-ionization is only slightly absorbed when passing through the $F_{2}$-layer, whereas the rays producing the $E$-ionization are completely absorbed when entering the $E$-layer. From this it follows that a variation in the pressure of the air in the $F_{2}$-layer will strongly influence the ion-production of the rays, as the spectral energy producing the ionization is in excess. For the $E$-layer, where the ionizing rays are completely absorbed when entering the layer, a variation of pressure (and therefore temperature) will be of minor importance, as the maximum ionization there will be determined by the limited energy of the ionizing rays.

Chapman has developed a theory according to which there is a stratification in the ionosphere; at heights above $200 \mathrm{~km}$. the oxygen molecules are dissociated and occur as atomic oxygen. There will therefore be different parts of the solar spectrum which produce the ionization in different levels, according to the different ionization potentials of the constituents. The ionization potentials of $\mathbf{N}_{2}$, $\mathrm{O}_{2}$ and $\mathrm{O}$ correspond to the bands from $766 \mathrm{~A}$., $730 \mathrm{~A}$. and $910 \mathrm{~A}$., and according to Chapman ${ }^{5}$ the energy available in the solar spectrum from $910 \mathrm{~A}$. 